\title{
Potentially Inappropriate Medication Use in Older Adults in the Preoperative Period: A Retrospective Study of a Noncardiac Surgery Cohort
}

\author{
Marie-France Forget ${ }^{1}\left[\right.$ Emily Gibson McDonald ${ }^{2,3,4} \cdot$ Astrid Bicamumpaka Shema $^{5} \cdot$ Todd Campbell Lee $^{2}$. \\ Han Ting Wang ${ }^{6}$
}

Published online: 18 April 2020

(c) The Author(s) 2020

\begin{abstract}
Background Few studies have evaluated the prevalence of potentially inappropriate medications (PIMs) and its association with postoperative outcomes in a geriatric population in the preoperative setting.

Objectives The purpose of this study was to evaluate the prevalence of PIMs in an older elective surgery population and to explore associations between PIMs and postoperative length of stay (LOS) and emergency department (ED) visits in the 90 days post hospital discharge, depending on frailty status.

Methodology We performed a retrospective cohort study of older adults awaiting major elective noncardiac surgery and undergoing an evaluation in the preoperative clinic at a tertiary academic center between 2017 and 2018. We identified PIMs using MedSafer, a software tool built to improve the safety of prescribing. Frailty status was assessed using the 7-point Clinical Frailty Scale. We estimated the association between PIMs and postoperative LOS and ED visits in the 90 days post hospital discharge.

Results The MedSafer software generated 394 recommendations on PIMs in 1619 medications for 252 patients. In total, 197 (78\%) patients had at least one PIM. The cohort included 138 (51\%) robust, 87 (32.2\%) vulnerable and $45(16.7 \%)$ frail patients. The association between PIMs and LOS was not significant for the robust and frail subgroups. For the vulnerable patients, every additional PIM increased LOS by $20 \%$ (incidence rate ratio 1.20 ; $95 \%$ confidence interval $0.90-1.44$; $p=0.089$ ) without reaching statistical significance. No association was found between PIMs and ED visits.

Conclusion PIMs identified by the MedSafer software were prevalent. Preoperative evaluation represents an opportunity to plan deprescribing of PIMs.
\end{abstract}

\section{Key Points}

Potentially inappropriate medications (PIMs) are prevalent in older adults undergoing elective noncardiac surgery.

Using MedSafer software in preoperative evaluations offers an opportunity to identify PIMs.

Electronic supplementary material The online version of this article (https://doi.org/10.1007/s40801-020-00190-y) contains supplementary material, which is available to authorized users.

Marie-France Forget

marie-france.forget@umontreal.ca

Extended author information available on the last page of the article

\section{Background and Objectives}

Polypharmacy, or taking multiple medications, is common in older adults and is an important public health matter. It is associated with an increasing number of comorbidities in the aging population $[1,2]$. The greater the number of prescriptions, the more likely there will be potentially inappropriate medications (PIMs) [3, 4]. PIMs are medications for which the risk of adverse events outweighs clinical benefits, especially when safer alternatives are available $[5,6]$. Few studies have evaluated the prevalence of PIMs in the preoperative setting and its association with postoperative outcomes [7-9]. One retrospective cohort study of 475 older patients awaiting oncological surgery showed a significant association between PIMs and the incidence of postoperative delirium (adjusted odds ratio [AOR] 5.53; 95\% confidence interval [CI] 2.03-15.05) [9]. One prospective study in 206 patients aged $>70$ years discharged from acute care 
identified frailty status as a predictor of PIMs at both admission (AOR 1.06; 95\% CI 1.01-2.37; $p<0.05$ ) and discharge (AOR 1.08; 95\% CI 1.06-2.36; $p<0.05$ ) [10]. Frailty is a clinical syndrome representing a reduced physiological reserve that carries an increased vulnerability for poor health outcomes [11]. Frailty is associated with adverse outcomes in the surgical setting [12]. The impact of PIMs might differ between frail and nonfrail older adults. In a retrospective study of 885 older women with prior admission for acute coronary syndromes (mean age $82.7 \pm$ standard deviation 2.7 years), the use of recommended medication was associated with an increase in the number of fall-related admissions (hazard ratio [HR] 2.57; 95\% CI 1.24-5.33). The association was stronger in frail older women only (HR 5.46; 95\% CI 1.34-22.3) [13].

Existing medication review processes in the preoperative setting are geared toward withholding potentially harmful medication for the perioperative period. This medication is usually resumed later on.

Therefore, our objective was to evaluate the prevalence of PIMs in an elderly elective surgery population presenting to the preoperative clinic and to explore any associations between PIMs and postoperative length of stay (LOS) and emergency department (ED) visits post hospital discharge according to frailty status.

\section{Methods and Analysis}

\subsection{Study Design}

We performed a retrospective cohort study. The analysis included a population of older adults awaiting elective surgery at Maisonneuve-Rosemont Hospital, a tertiary academic center in Montréal, Québec, Canada, between January 2017 and January 2018 who participated in a prospective study assessing the impact of frailty on postoperative outcomes. The inclusion criteria were age $\geq 65$ years at the time of preoperative evaluation, awaiting a major elective surgery with an expected hospitalization stay of $>24 \mathrm{~h}$, and undergoing an evaluation in the preoperative clinic of Maisonneuve-Rosemont Hospital. We excluded those who were unable to consent or be contacted prior to surgery and those for whom a medication list was unavailable. Consent was obtained before we assessed frailty state. Consent was obtained at the time of the pre-operative evaluation and then patients were contacted via telephone to complete data collection and specifically a frailty evaluation. Eligible surgeries included orthopedic surgery (hip and knee arthroplasty, sarcoma resection), vascular surgery (aortic, aortobifemoral, iliofemoral bypass, carotid endarterectomy) and general surgery (hernia repair, hemicolectomy, colostomy, ileostomy closure, low anterior rectum resection). All patients were seen by a nurse and an internist as part of their routine evaluation. Medication lists were obtained from the patient's community pharmacy and reviewed by the nurse and internist for preoperative management. A formal review process of PIMs and deprescribing was not part of the routine assessment in our center. This study was approved by the Research Ethics Board of Maisonneuve-Rosemont Hospital.

\subsection{Data Collection}

The following variables, collected in the preoperative period, were available in the existing database: demographics, comorbidities, Charlson comorbidity score (computed from the known variables of the Charlson Comorbidity Index, except age [14]), creatinine level at the time of preoperative assessment, American Society of Anesthesiologists score [15], surgical specialty and frailty score. We also presented the most frequent comorbidities found in our cohort. Frailty assessment was performed by a trained research assistant using the 7-point Clinical Frailty Scale, a validated and reliable frailty instrument [11] that considers whether patients' chronic illnesses are under control and their independence in activities of daily living and instrumental activities of daily living. Patients were classified on a numerical scale (1-7), where scores of 1-3 indicated robust, 4 indicated vulnerable, and $\geq 5$ indicated frail. Vulnerability is defined in the Clinical Frailty Scale as being slowed up but not dependent on others to accomplish daily activities. Frailty was assessed during the preoperative visit through a semi-structured interview.

We collected medication data using the patient's medication list provided at the time of preoperative evaluation. Only chronic medications were considered and were classified according to the Anatomical Therapeutic Chemical classification system. We excluded ophthalmological drops, dermatological preparations and short-term treatments such as antibiotics.

\subsubsection{Identification of Potentially Inappropriate Medications (PIMs)}

We identified PIMs using MedSafer, which is a Canadian software tool built to improve the safety of prescribing. It is an electronic application that identifies PIMs in older adults based on existing evidence-based lists [16]. It includes all of the recommendations for deprescribing contained in the Beers Criteria [17], the Screening Tool of Older People's Prescriptions (STOPP) [18], Choosing Wisely [19] and some emerging evidence. The development of the software has 
been previously described [20]. MedSafer identifies specific triggering conditions, relevant drug-drug interactions and drug-disease interactions using patients' comorbidities and usual home medications. For each PIM, a recommendation and rationale to stop or reconsider the medication is generated, with a tapering regimen when necessary (Electronic Supplementary Material [ESM] 1). Each recommendation is assigned one of three stopping priorities:

1. High risk: should be reconsidered right away and stopped as soon as possible if clinically indicated.

2. Intermediate risk: deprescribing should factor in what the prescriber knows about the patient and needs to balance the risks and benefits.

3. Low risk: stopping is recommended mostly because of a lack of evidence for effectiveness. These medications add to pill burden.

\subsubsection{Clinical Outcomes}

Our primary outcome was postoperative hospital LOS, and our secondary outcome was ED visit in the 90 days following hospital discharge.

\subsection{Statistical Methods}

We used SPSS Statistics (version 25) for analysis. We reported descriptive statistics for the complete cohort of surgical patients evaluated at our preoperative clinic included in the prospective study. Kolmogorv-Smirnov or Shapiro-Wilk tests were used to test for normality distribution of continuous variables, which were then reported as mean or median with their central tendency distribution. Categorical variables were reported as proportions. We used two independent sample $t$ tests and the Wilcoxon-Mann-Whitney test to compare continuous variables and the Chi-squared and Fisher's exact test for categorial variables for comparison between two groups (Kruskal-Wallis and one-way analysis of variance for comparisons between three groups). We reported the total number of PIM recommendations for the surgical cohort and the percentage of PIM recommendations per priority 1,2 and 3 .

We explored the association between the number of PIMs and LOS using multivariate negative binomial regression and the association between the number of PIMs and ED visits using multivariate logistic regression. The primary analyses were stratified by frailty status (robust, vulnerable or frail). Sensitivity analysis were completed with stratification by surgery specialty (orthopedic and nonorthopedic). The following a priori covariates were included in our models: age, sex, Charlson comorbidity score and surgery specialty. These analyses were only possible for patients with at least one chronic medication who underwent surgery.

\section{Results}

\subsection{Patients}

A total of 300 patients underwent frailty assessment in our preoperative clinic, and 270 patients underwent surgery (ESM 2). The median age of the surgical cohort was 73 years (interquartile range [IQR] 69-76), and 145 (54\%) patients were female (Table 1). Patients underwent orthopedic surgery $(n=165[61 \%])$, general surgery $(n=68[25 \%])$ or vascular surgery $(n=37[14 \%])$. The median time between preoperative visit and surgery was 63 days (IQR 35.8-90). The most prevalent comorbidities were hypertension (190 [70\%]), dyslipidemia (161 [60\%]) and diabetes (75 [27.8\%]). According to the Clinical Frailty Scale, 138 (51\%) patients were considered robust, 87 (32\%) vulnerable and 45 (17\%), frail.

\subsection{Medication}

A total of 1668 individual prescriptions were recorded for 270 patients. After excluding 49 ophthalmological drops or dermatological preparations, 1619 prescriptions were considered for analysis. The median number of prescriptions per patients was 6 (IQR 3-8). Only 18 (6.7\%) patients did not take any chronic medications before surgery, whereas 175 (64.8\%) patients met our definition of polypharmacy (five or more medications). Medication use was similar between patients who did or did not undergo surgery (ESM 3).

The three most common medication categories were cardiovascular (519 prescriptions [32\%]), alimentation tract and metabolism (465 prescriptions [29\%]) and nervous system (181 prescriptions [11\%]) (ESM 4). The cardiovascular category mainly comprised lipid-modifying agents (160 [32\%]), antihypertensives (146 [28\%]) and agents acting on the renin-angiotensin system (114 [22\%]). The alimentation tract and metabolism included $\mathrm{H}_{2}$-receptor antagonists and proton pump inhibitors (125 [27\%]) and vitamins (117 [25\%]). The nervous system category contained mostly antidepressants (69 [38\%]), antiepileptics (50 [28\%]), psycholeptics (20 [11\%]) and opioids (21 [12\%]).

We processed the medications of 252 patients who took at least one medication before surgery in the MedSafer software. It generated 394 recommendations on PIMs for 197 (78\%) patients. Only 55 (22\%) patients had no PIMs. Patients with PIMs were more frequently female and more frequently frail (ESM 5). The median number of recommendations per patient was 1 (IQR 1-2). High-risk medications were observed in $60(22.2 \%)$ patients. The priority 1 recommendations $(n=71[18 \%])$ mostly concerned benzodiazepines $(n=15[21 \%])$, sulfonylureas $(n=10[14 \%])$, dual antithrombotic therapy $(n=8[11 \%])$ and opiates for 
Table 1 Patient characteristics and comparisons between surgical and nonsurgical cohorts

\begin{tabular}{|c|c|c|c|c|}
\hline Characteristics & Total cohort & Surgical cohort & No surgery cohort & $p$ value \\
\hline Patients, $N$ & 300 & 270 & 30 & \\
\hline Age, years & $72(69-76)$ & $73(69-76)$ & $74(68-77)$ & 0.184 \\
\hline \multicolumn{5}{|l|}{ Sex } \\
\hline Female & $159(53)$ & $145(53.7)$ & $14(46.7)$ & 0.464 \\
\hline \multicolumn{5}{|l|}{ Most common comorbidities } \\
\hline Hypertension & $211(70.3)$ & $190(70.4)$ & $21(70)$ & 0.966 \\
\hline Dyslipidemia & $178(59.3)$ & $161(59.6)$ & $17(57)$ & 0.754 \\
\hline Diabetes & $82(27.3)$ & $75(27.8)$ & $7(23.3)$ & 0.604 \\
\hline Myocardial infarction & $75(25)$ & $63(23.3)$ & $12(40)$ & 0.046 \\
\hline Chronic kidney disease & $65(21.7)$ & $58(21.5)$ & $7(23.3)$ & 0.815 \\
\hline Peripherical artery disease & $43(14.3)$ & $37(13.7)$ & $6(20)$ & 0.407 \\
\hline Chronic obstructive pulmonary disease & $41(13.7)$ & $37(13.7)$ & $4(13.3)$ & 1.000 \\
\hline Cancer & $36(12)$ & $30(11.1)$ & $6(20)$ & 0.230 \\
\hline Cerebrovascular disease & $32(10.7)$ & $30(11.1)$ & $2(6.7)$ & 0.754 \\
\hline Connective tissue disease & $14(4.7)$ & $13(4.8)$ & $1(3.3)$ & 1.000 \\
\hline Congestive heart failure & $11(3.7)$ & $7(2.6)$ & $4(13.3)$ & 0.016 \\
\hline Charlson comorbidity Score ${ }^{a}$ & $1(0-3)$ & $1(0-3)$ & $2(0-3)$ & 0.321 \\
\hline \multicolumn{5}{|l|}{ Type of surgery planned } \\
\hline Orthopedics & $179(59.7)$ & $165(61.1)$ & $14(46.7)$ & 0.304 \\
\hline General surgery & $78(26)$ & $68(25.2)$ & $10(33.3)$ & \\
\hline Vascular surgery & $43(14.3)$ & $37(13.7)$ & $6(20)$ & \\
\hline \multicolumn{5}{|l|}{ Clinical Frailty Scale } \\
\hline Robust & 149 (49.7) & $138(51.1)$ & $11(36.7)$ & 0.028 \\
\hline Vulnerable & $95(31.7)$ & $87(32.2)$ & $8(26.7)$ & \\
\hline Frail & $56(18.7)$ & $45(16.7)$ & $11(36.7)$ & \\
\hline No medication & $19(6.3)$ & $18(6.7)$ & $1(3.3)$ & 0.705 \\
\hline 5 or more medications & $194(64.7)$ & $175(64.8)$ & $19(63.3)$ & 0.872 \\
\hline 9 or more medications & $79(26.3)$ & $71(26.3)$ & $8(26.7)$ & 0.965 \\
\hline
\end{tabular}

Data are presented as median (interquartile range) or $N(\%)$ unless otherwise indicated

${ }^{a}$ Charlson Comorbidity Score $=$ Comorbidities of the Charlson Comorbidity Index chronic noncancer pain ( $n=5$ [7\%]) (ESM 6). Priority 2 recommendations ( $n=216[55 \%])$ mostly concerned chronic proton pump inhibitor therapy $(n=115[53 \%])$ and pregabalin $(n=40[19 \%])$. The priority 3 recommendations were related to medications with low-quality or absent evidence of effectiveness, such as docusate for constipation $(n=107$ [27\%]).

\subsection{Association of PIMs with Length of Stay and Emergency Department Visits in the 90 Days Following Discharge}

The median LOS was 4 days (IQR 2-7). After stratification by frailty status (ESM 7), frail patients had a longer median LOS (6.5 [IQR 3-9]) than did robust (4 days [IQR 2-6]) and vulnerable (5 days [IQR 2-7]) patients $(p=0.002)$. Frail and vulnerable patients had more PIMs than robust patients (2 PIMs [IQR 1-3], 2 PIMs [IQR 1-3], 1 PIM [IQR 0-2], respectively; $p=0.000$ ). The Charlson comorbidity score was comparable in the three groups (1 [IQR 0-3], 2 [IQR 1-3], 2 [IQR 0-3], respectively; $p=0.517$ ). The association between the number of PIMs and LOS was not significant for the subgroup of robust and frail patients (Table 2). For vulnerable patients, an increase in LOS with a higher number of PIMs was almost statistically significant. For every additional PIM, there was a $20 \%$ increase in LOS (incidence rate ratio [IRR] $1.20 ; 95 \% \mathrm{CI} 0.98-1.44 ; p=0.089$ ). For our sensitivity analysis, orthopedic and nonorthopedic patients had similar LOS (4 days [IQR 3-7] vs. 4 [1-7]; $p=0.575$ ). For nonorthopedic patients, PIMs were associated with a statistically significant increase in LOS. For every additional PIM, there was a $28 \%$ increase in LOS (IRR 1.28 ; 95\% CI $1.02-1.61 ; p=0.039)$ (ESM 8). In total, $40(15 \%)$ patients visited the $\mathrm{ED}$ in the 90 days following hospital discharge (median time from discharge to ED visit 13.5 days [IQR 
Table 2 Multivariable negative binomial regression of factors associated with length of stay, stratified by frailty status (patient of the surgical cohort taking one or more medication)

\begin{tabular}{|c|c|c|c|c|c|c|}
\hline \multirow[t]{2}{*}{ Variable } & \multicolumn{2}{|c|}{ Robust group ( $n=123$ ) } & \multicolumn{2}{|c|}{ Vulnerable group $(n=85)$} & \multicolumn{2}{|c|}{ Frail group $(n=44)$} \\
\hline & IRR (95\% CI) & $p$ value & IRR $(95 \%$ CI $)$ & $p$ value & IRR $(95 \%$ CI $)$ & $p$ value \\
\hline Age & $1.03(0.99-1.07)$ & 0.153 & $1.01(0.97-1.06)$ & 0.561 & $0.99(0.93-1.06)$ & 0.811 \\
\hline Charlson Comorbidity Score ${ }^{a}$ & $1.02(0.88-1.20)$ & 0.761 & $0.88(0.75-1.02)$ & 0.098 & $0.89(0.71-1.12)$ & 0.322 \\
\hline \multicolumn{7}{|l|}{ Surgery specialty } \\
\hline Orthopedics & 1 & & 1 & & 1 & \\
\hline General surgery & $1.60(0.93-2.75)$ & 0.089 & $1.06(0.57-1.98)$ & 0.666 & $1.03(0.37-2.87)$ & 0.949 \\
\hline Vascular surgery & $1.40(0.69-2.83)$ & 0.348 & $1.51(0.75-3.01)$ & 0.224 & $0.59(0.17-2.01)$ & 0.409 \\
\hline \multicolumn{7}{|l|}{ Sex } \\
\hline Female & 1 & & 1 & & 1 & \\
\hline Male & $1.02(0.65-1.60)$ & 0.919 & $0.85(0.50-1.44)$ & 0.544 & $0.63(0.31-1.28)$ & 0.628 \\
\hline Number of PIMs & $1.10(0.89-1.67)$ & 0.327 & $1.20(0.98-1.44)$ & 0.089 & $0.96(0.79-1.17)$ & 0.692 \\
\hline
\end{tabular}

$C I$ confidence interval, IRR incidence relative ratio, PIMs potentially inappropriate medications

${ }^{\mathrm{a}}$ Charlson Comorbidity Score = comorbidities of the Charlson Comorbidity Index

6.25-32.75]). The number of PIMs was not associated with ED visits for any of the frailty groups (Table 3).

\section{Discussion}

PIMs identified by the MedSafer software were prevalent in our cohort of older adults awaiting elective surgery. An increasing number of PIMs in vulnerable older adults seemed to be associated with a longer hospital LOS in our adjusted model but did not reach statistical significance.

The prevalence of PIMs in our cohort was higher than in past studies. In a 2016 retrospective study of a colorectal cancer surgery population ( $n=7279$, aged $\geq 75$ years), the prevalence of PIMs was 22.5\% [9]. Their definition of PIMs was more selective than the MedSafer software and included fewer medication class (psycholeptics, analgesics, anticholinergics, opioids, antidepressants, non-steroid anti-inflammatories).

In our study, the vulnerable and frail populations had more PIMs than the robust group even when the disease burden, as measured by the Charlson comorbidity score, was comparable. Presentation of illness differs between older and younger adults, with older patients showing more atypical signs and symptoms, potentially requiring more medication and therefore having an increased pill burden [21-23]. Unusual symptoms might result in more prescriptions and, indeed, probably more PIMs. This is the so-called

Table 3 Multivariable logistic regression of factors associated with emergency department visits, stratified by frailty status

\begin{tabular}{|c|c|c|c|c|c|c|}
\hline \multirow[t]{2}{*}{ Factors } & \multicolumn{2}{|c|}{ Robust group $(N=11 / 123)$} & \multicolumn{2}{|c|}{ Vulnerable group $(N=20 / 85)$} & \multicolumn{2}{|c|}{ Frail group $(N=9 / 44)$} \\
\hline & OR $(95 \mathrm{CI})$ & $p$ value & OR (95 CI) & $p$ value & OR (95 CI) & $p$ value \\
\hline Age & $0.98(0.86-1.11)$ & 0.719 & $1.00(0.89-1.12)$ & 0.933 & $0.97(0.84-1.13)$ & 0.690 \\
\hline Charlson comorbidity score ${ }^{a}$ & $1.08(0.75-1.55)$ & 0.683 & $1.97(1.23-3.16)$ & 0.005 & $1.00(0.59-1.70)$ & 0.991 \\
\hline \multicolumn{7}{|l|}{ Surgery specialty } \\
\hline Orthopedics & 1 & & 1 & & 1 & \\
\hline General surgery & $0.20(0.05-0.80)$ & 0.024 & $1.31(0.22-7.75)$ & 0.764 & $0.24(0.26-2.18)$ & 0.202 \\
\hline Vascular surgery & $0.12(0.02-0.74)$ & 0.022 & $0.61(0.13-2.97)$ & 0.538 & b & $\mathrm{b}$ \\
\hline \multicolumn{7}{|l|}{ Sex } \\
\hline Female & 1 & & 1 & 0.966 & 1 & 0.900 \\
\hline Male & $2.43(0.71-8.39)$ & 0.159 & $0.97(0.25-3.71)$ & & $1.12(0.19-6.49)$ & \\
\hline Number of PIMs & $0.95(0.53-1.71)$ & 0.857 & $0.60(0.31-1.14)$ & 0.119 & $1.51(0.90-2.52)$ & 0.119 \\
\hline
\end{tabular}

$C I$ confidence interval, $E D$ emergency department, $O R$ odds ratio, PIM potentially inappropriate medication

${ }^{a}$ Charlson Comorbidity Score $=$ comorbidities of the Charlson Comorbidity Index

${ }^{b}$ No ED visit in older adults who underwent vascular surgery and are in the frail group 
prescribing cascade, whereby one medication is added to treat the symptom that arises from another medication (e.g., an antihypertensive therapy to treat hypertension from an NSAID) [24]. Having more PIMs may be a measure of underlying geriatric susceptibility [25].

A positive association was close to being statistically significant between longer hospital LOS and increasing PIMs for vulnerable patients. Vulnerable older adults in a transitional state between robust and frail may be at higher risk of adverse events from PIMs and may also benefit most from deprescribing. For frail patients, the underlying frailty burden and functional decline might outweigh the impact of PIMs. Similarly, in a retrospective cohort study in a large elective noncardiac surgery geriatric population ( $n=266,499$, aged $>66$ years), the association between polypharmacy (five or more medications) and death within 90 days was not significant in patients with a higher frailty status [26]. Finally, in robust patients, PIMs might not significantly affect their capacity to cope with disease and stressors such as surgery.

The MedSafer software identifies three stopping priorities. It allows the clinician to choose PIMs to focus on-either those that could cause harm or medication that impacts the pill burden or raises the total medication cost. Those last aspects are usually not part of the medication review process in the preoperative setting. Since pill burden impacts well-being and quality of life [27, 28], it stands to reason that withdrawing medications lacking evidence for effectiveness could improve patient outcomes in the postoperative period. Beyond usual practice, medication review could be a good opportunity to optimize medication effectiveness and maybe reduce future harm with a standardized deprescribing process. In the outpatient setting, medication review has been shown to reduce hospital visits and delay the time to first ED visit after discharge [29]. To our knowledge, no such data exist in the surgical literature. However, the feasibility of such an intervention in the perioperative setting is unclear since deprescribing requires time, patient engagement and close follow-up [30]. For example, general practitioners identified barriers such as a lack of knowledge about withdrawal side effects and discomfort about changing medications prescribed by another physician [31]. Whether internists or surgeons experience the same reluctance needs to be studied.

Our study had several limitations. Generalizability is a concern as this study took place in a single academic center. We were most likely not powered to show an association between PIMs and LOS or ED visits given the overall low prevalence of high-risk PIMs and the small proportion of patients who returned to the hospital in the 90 days post surgery. Our sample sizes, particularly the frail and the vascular surgery groups, were limited. Therefore, we could not explore how frailty status affected LOS. It could be a confounder and an effect modifier. Also, MedSafer does not highlight the absence of proper medications for any given conditions and does not incorporate the Screening Tool to Alert to Right Treatment (START) rules (e.g., angiotensinconverting enzyme inhibitor for left ventricular dysfunction). We were unable to assess patients' compliance with medication and or consider over-the-counter PIMs. Peri- and postoperative medication could have been associated with the outcomes but was not assessed.

The strengths of this study included the novel use of comprehensive electronic software to identify PIMs in patients undergoing a preoperative assessment from different surgical specialties. To our knowledge, this is the first study to assess opportunities for deprescribing in the preoperative setting. The use of MedSafer has previously been shown to decrease PIMs in the acute care setting by prioritizing PIMs for deprescribing and providing a rationale and withdrawal recommendations to clinicians. With that information in hand, deprescribing could be easier to implement in the preoperative period and beyond. Another strength of the study was that we integrated the concept of frailty in the association between PIMs and postoperative outcomes. It allowed us to tease out vulnerable older adults as a group to target for deprescribing.

\section{Conclusion}

We found that PIMs were prevalent in the preoperative setting and, in a larger cohort of patients, may influence LOS for vulnerable older adults. Preoperative evaluation represents an opportunity to deprescribe PIMs or plan for deprescribing after surgery. Future prospective interventional studies in the preoperative setting need to assess the feasibility and acceptability of deprescribing using MedSafer and the impacts on postoperative outcomes.

Author Contributions Conceptualization: EGM, TCL, HTW. Methodology: M-FF, EGM. Formal analysis and investigation: M-FF, ABS, HTW. Original draft preparation: M-FF. Review and editing: M-FF, EGM, ABS, TCL, HTW. Resources: M-FF, ABS. Supervision: TCL, HTW.

Data Availability The datasets analyzed during the current study are available from the corresponding author on reasonable request.

\section{Compliance with Ethical Standards}

Funding No sources of funding were used to conduct this study or prepare this manuscript. 
Conflict of interest Marie-France Forget, Emily Gibson McDonald, Astrid Bicamumpaka Shema, Todd Campbell Lee, and Han Ting Wang have no conflicts of interest that are directly relevant to the content of this article.

Open Access This article is licensed under a Creative Commons Attribution-NonCommercial 4.0 International License, which permits any non-commercial use, sharing, adaptation, distribution and reproduction in any medium or format, as long as you give appropriate credit to the original author(s) and the source, provide a link to the Creative Commons licence, and indicate if changes were made. The images or other third party material in this article are included in the article's Creative Commons licence, unless indicated otherwise in a credit line to the material. If material is not included in the article's Creative Commons licence and your intended use is not permitted by statutory regulation or exceeds the permitted use, you will need to obtain permission directly from the copyright holder.To view a copy of this licence, visit http://creativecommons.org/licenses/by-nc/4.0/.

\section{References}

1. Barnett K, Mercer SW, Norbury M, et al. Epidemiology of multimorbidity and implications for health care, research, and medical education: a cross-sectional study. Lancet. 2012;380(9836):37-433.

2. Kaufman DW, Kelly JP, Rosenberg L, et al. Recent patterns of medication use in the ambulatory adult population of the United States: the Slone survey. JAMA. 2002;287(3):337-44.

3. Aparasu RR, Mort JR. Inappropriate prescribing for the elderly: beers criteria-based review. Ann Pharmacother. 2000;34(3):338-46.

4. Santos AP, da Silva DT, dos Santos Junior GA, et al. Evaluation of the heterogeneity of studies estimating the association between risk factors and the use of potentially inappropriate drug therapy for the elderly: a systematic review with meta-analysis. Eur J Clin Pharmacol. 2015;71(9):1037-50.

5. Motter FR, Fritzen JS, Hilmer SN, et al. Potentially inappropriate medication in the elderly: a systematic review of validated explicit criteria. Eur J Clin Pharmacol. 2018;74(6):679-700.

6. Muhlack DC, Hoppe LK, Weberpals J, et al. The association of potentially inappropriate medication at older age with cardiovascular events and overall mortality: a systematic review and meta-analysis of cohort studies. J Am Med Dir Assoc. 2017;18(3):211-20.

7. Choi KS, Jeong YM, Lee E, et al. Association of pre-operative medication use with post-surgery mortality and morbidity in oncology patients receiving comprehensive geriatric assessment. Aging Clin Exp Res. 2018;30:1177-85.

8. Samuelsson KS, Egenvall M, Klarin I, et al. Inappropriate drug use in elderly patients is associated with prolonged hospital stay and increased postoperative mortality after colorectal cancer surgery: a population-based study. Colorectal Dis. 2016;18(2):155-62.

9. Jeong YM, Lee E, Kim KI, et al. Association of pre-operative medication use with post-operative delirium in surgical oncology patients receiving comprehensive geriatric assessment. BMC Geriatr. 2016;16:134

10. Herr M, Sirven N, Grondin H, et al. Frailty, polypharmacy, and potentially inappropriate medications in old people: findings in a representative sample of the French population. Eur J Clin Pharmacol. 2017;73(9):1165-72.

11. Rockwood K, Song X, MacKnight C, et al. A global clinical measure of fitness and frailty in elderly people. CMAJ. 2005;173(5):489-95.

12. Okabe H, Ohsaki T, Ogawa K, et al. Frailty predicts severe postoperative complications after elective colorectal surgery. Am J Surg. 2019;217(4):677-81.

13. Peeters G, Tett SE, Hollingworth SA, et al. Associations of Guideline Recommended Medications for acute coronary syndromes with fall-related hospitalizations and cardiovascular events in older women with ischemic heart disease. J Gerontol A Biol Sci Med Sci. 2017;72(2):259-65.

14. Charlson ME, Pompei P, Ales KL, et al. A new method of classifying prognostic comorbidity in longitudinal studies: development and validation. J Chronic Dis. 1987;40(5):373-83.

15. ASA House of Delegates/Executive Committee. ASA Physical Status Classification System. 2014 October 15, 2014. https://www. asahq.org/standards-and-guidelines/asa-physical-status-classifica tion-system. Accessed 25 Apr 2019.

16. McDonald EG, Wu PE, Rashidi B, et al. The MedSafer Study: a controlled trial of an electronic decision support tool for deprescribing in acute care. J Am Geriatr Soc. 2019;67(9):1843-50.

17. American Geriatrics Society 2015 Beers Criteria Update Expert Panel. American Geriatrics Society 2015 Updated Beers Criteria for potentially inappropriate medication use in older adults. J Am Geriatr Soc. 2015;63(11):2227-46.

18. O'Mahony D, O'Sullivan D, Byrne S, et al. STOPP/START criteria for potentially inappropriate prescribing in older people: version 2 . Age Ageing. 2015;44(2):213-8.

19. Levinson W. Choosing Wisely Canada Recommendations. https:// choosingwiselycanada.org/recommendations/. Accessed 12 Nov 2018.

20. McDonald EG, Wu PE, Rashidi B, Forster AJ, Huang A, Pilote L, et al. The MedSafer Study: a controlled trial of an electronic decision support tool for deprescribing in acute care. J Am Geriatr Soc. 2019;67(9):1843-50.

21. Breining A, Negers A, Mora L, Moisi L, Golmard JL, Cohen A, et al. Determinants of clinical presentation on outcomes in older patients with myocardial infarction: atypical presentation in elderly with MI. Geriatr Gerontol Int. 2018;18(12):1591-6.

22. Daoust R, Paquet J, Piette E, et al. Impact of age on pain perception for typical painful diagnoses in the Emergency Department. J Emerg Med. 2016;50(1):14-20.

23. Limpawattana P, Phungoen P, Mitsungnern T, Laosuangkoon W, Tansangworn N. Atypical presentations of older adults at the emergency department and associated factors. Arch Gerontol Geriatr. 2016;62:97-102.

24. McIsaac DI, Wong CA, Diep D, van Walraven C. Association of preoperative anticholinergic medication exposure with postoperative healthcare resource use and outcomes: a population-based cohort study. Ann Surg. 2019;270(6):1049-57.

25. Hofman MR, van den Hanenberg F, Sierevelt IN, et al. Elderly patients with an atypical presentation of illness in the emergency department. Neth J Med. 2017;75(6):241-6.

26. McIsaac DI, Wong CA, Bryson GL, et al. Association of polypharmacy with survival, complications, and healthcare resource use after elective noncardiac surgery: a population-based cohort study. Anesthesiology. 2018;128(6):1140-50.

27. Mohammed MA, Moles RJ, Chen TF. Medication-related burden and patients' lived experience with medicine: a systematic review and metasynthesis of qualitative studies. BMJ Open. 2016;6(2): $\mathrm{e} 010035$.

28. Olsson IN, Runnamo R, Engfeldt P. Medication quality and quality of life in the elderly, a cohort study. Health Qual Life Outcomes. 2011;9:95.

29. Gillespie U, Alassaad A, Henrohn D, et al. A comprehensive pharmacist intervention to reduce morbidity in patients 80 years or older: a randomized controlled trial. Arch Intern Med. 2009;169(9):894-900.

30. Frank C, Weir E. Deprescribing for older patients. CMAJ. 2014;186(18):1369-76.

31. Djatche L, Lee S, Singer D, et al. How confident are physicians in deprescribing for the elderly and what barriers prevent deprescribing? J Clin Pharm Ther. 2018;43(4):550-5. 


\section{Affiliations}

\section{Marie-France Forget ${ }^{1}$ (D) Emily Gibson McDonald ${ }^{2,3,4} \cdot$ Astrid Bicamumpaka Shema $^{5} \cdot$ Todd Campbell Lee $^{2}$. Han Ting Wang ${ }^{6}$}

1 Division of Geriatric Medicine, Department of Medicine, Centre Hospitalier de l'Université de Montréal, Montreal, QC, Canada

2 Division of General Internal Medicine, Department of Medicine, McGill University Health Center, Montreal, QC, Canada

3 Center for Health Outcomes Research and Evaluation, Research Institute-McGill University Health Center, Montreal, QC, Canada
4 Clinical Practice Assessment Unit, Department of Medicine, McGill University Health Center, Montreal, QC, Canada

5 Université de Montréal, Montreal, QC, Canada

6 Division of Internal and Critical Care Medicine, Department of Medicine, Hôpital Maisonneuve-Rosemont, Université de Montréal, Montreal, QC, Canada 